\section{$\underset{\substack{\text { hommes } \\ \text { \& migrations }}}{ }$}

\section{Hommes \& migrations}

Revue française de référence sur les dynamiques

migratoires

\section{$1299 \mid 2012$}

Musulmanes et féministes en Grande-Bretagne

\title{
Django Drom
}

Rencontre avec Tony Gatlif

\section{François Bensignor}

\section{(2) OpenEdition \\ 12 Journals}

Édition électronique

URL : http://journals.openedition.org/hommesmigrations/1432

DOI : 10.4000/hommesmigrations. 1432

ISSN : 2262-3353

Éditeur

Musée national de l'histoire de l'immigration

Édition imprimée

Date de publication : 1 septembre 2012

Pagination : 142-147

ISSN : 1142-852X

\section{Référence électronique}

François Bensignor, «Django Drom », Hommes \& migrations [En ligne], 1299 | 2012, mis en ligne le 29 mai 2013, consulté le 22 septembre 2020. URL : http://journals.openedition.org/hommesmigrations/ 1432 ; DOI : https://doi.org/10.4000/hommesmigrations.1432

Ce document a été généré automatiquement le 22 septembre 2020.

Tous droits réservés 


\title{
Django Drom
}

\author{
Rencontre avec Tony Gatlif
}

François Bensignor

\section{NOTE DE L'ÉDITEUR}

Django Reinhardt - Swing de Paris : exposition au Musée de la musique jusqu'au 20 janvier 2013.

Chacune de vos œuvres, film ou spectacle, est un hommage au peuple rom, qu'il s'agisse des Tsiganes d'Europe de l'Est, des Gitans ou des Manouches. Pourquoi tous ces hommages?

Tony Gatlif : Parce que avec toutes les casseroles que traîne le peuple rom à longueur d'année en Europe, dix hommages, ce n'est pas assez, il en faudrait mille ! On est très peu à rendre hommage à ce peuple. Heureusement, le public des concerts, des salles de cinéma, est là nombreux en France et dans le monde... Beaucoup de gens aiment les films sur les Tsiganes comme leurs musiques. Ce public rend hommage à ce peuple en allant au concert ou voir mes films. Mais à l'inverse, toutes les politiques mises en œuvre en Europe le pourchassent.

Pendant deux étés consécutifs, les Roms ont été au centre de l'actualité en France. Quel est votre point de vue sur la question?

T. G. : Je me suis exprimé sur le discours de Sarkozy à Grenoble, parce qu'il était d'une terrible violence. Il a parlé d'une façon négative du peuple rom et restera dans les mémoires à cause de cela. Je me suis mobilisé avec les Manouches, ceux qui parlent le romanès. Eux sont directement concernés, alors que moi, je ne suis pas un représentant des Roms. Je n'ai jamais été leur porte-parole et ne voudrai jamais l'être. Je suis né d'un père kabyle et d'une mère gitane, mais je ne représente pas la communauté rom. Je suis un cinéaste, un artiste, et comme mes films sont connus dans le monde entier, je peux parler. Mais je ne vais pas parler de ce que je ne connais pas, ni me lancer à faire de la politique. Ce n'est pas mon métier. J'imagine mal un Corse parlant sa langue être représenté par quelqu'un qui ne parle pas cette langue. 
C'est une chose impensable chez les Arméniens, chez les Arabes ou tout peuple que ce soit ayant sa propre langue. Alors, comment se fait-il que des gens qui ne parlent pas la langue rom, qui ne sont pas nés roms, soient justement ceux qui parlent pour les Roms? Voilà trente-cinq ans que je tourne des films autour des Roms. J'ai connu tous leurs représentants. En voyageant, j'ai rencontré des Roms en Roumanie, en Hongrie, au Kosovo, de jeunes intellectuels brillants. J'en connais chez les Manouches, chez les Gitans d'Espagne : ces gens ont fait des choses magnifiques en faveur des Roms de Roumanie, de Bulgarie, du Kosovo, en voyant que le monde entier s'acharnait contre eux jusqu'en Amérique latine. Ils se mobilisent pour défendre leur "romanité". En Espagne, où je suis allé avec eux pour protester à la Puerta del Sol à Madrid, ils ne disent plus qu'ils sont gitans, mais roms. Pourtant ici en France, je suis complètement désemparé de constater que les Roms que je connais pour être des personnalités brillantes ne sont pas acceptés dans les discussions avec le gouvernement concernant la politique à l'égard des Roms. Je n'en veux à personne, mais je pense que lorsqu'il s'agit des politiques qui les concernent, les Roms doivent être consultés.

Vous dites que vous ne voulez pas faire de politique, pourtant, avec votre film Liberté, vous avez posé une question historique qui a son importance dans le débat politique...

T. G. : Oui, mais ce que je dis là concerne la situation actuelle. Les Roms ont plein d'amis qui ne sont pas roms et qui font des choses superbes. Mais la question que je pose, c'est: Où sont les Manouches et les autres Roms quand il s'agit des politiques qui les concernent directement? Pourquoi les Roms n'ont-ils pas été invités à parler avec le gouvernement? Je considère que nous avons un gouvernement humain. Je l'ai soutenu et le soutiens toujours. Mais je ne comprends pas que ce malaise persiste et qu'on ne laisse pas les Roms s'exprimer en leur propre nom. Le vrai problème en France, comme en Allemagne, est affaire de respect. Donner du travail aux Roms ne sert à rien tant qu'on ne les respecte pas. Et la première marque de respect serait d'abord de leur donner la parole. Je ne critique pas les associations. Elles font un travail fantastique pour aider les Roms. Mais elles ne sont pas qualifiées pour défendre leur existence. C'est à eux de parler.

Vous êtes très préoccupé par la disparition de la culture tsigane. Qu'est-ce qui pourrait en conserver l'esprit dans notre monde contemporain?

T. G. : Je m'emballe parce que je constate qu'on est en train de tuer la culture rom. On voudrait faire entrer ces gens dans un même moule, les intégrer... Mais les "villages d'insertion" ressemblent à des camps, avec leurs vigiles, leurs chiens, les numéros à présenter, la porte fermée derrière chacun et close à toute personne non invitée... Pour une population habituée à vivre sa liberté, cela ressemble beaucoup à de l'enfermement. Et je vois peu de différences entre un "village d'insertion" et une maison de correction. Mais admettons que ce mode d'insertion fonctionne, quel est l'avenir de ces populations? Le même que celui des familles émigrées d'Afrique du Nord dans les bidonvilles de Nanterre, qui ont été relogées dans des cités où leurs enfants se retrouvent sans travail, coupés de leurs racines et incapables de s'exprimer face à une société qui les rejette?

Selon vous, l'engouement pour les musiques tsiganes des Balkans en Europe de l'Ouest a-til contribué à changer le regard porté sur les Roms?

T. G. : Oui, il a servi à changer la mentalité d'une certaine frange de jeunes, ceux dont je parlais, qui remplissent les salles de cinéma et de concerts... L'ouverture de l'Europe a apporté pas mal de problèmes. Les Roms, d'abord décimés par les nazis et 
maltraités dans les pays d'Europe orientale, se sont retrouvés enfermés dans le bloc de l'Est, sous les dictatures de Ceausescu ou de Tito. Et quand l'Europe s'est ouverte, on a découvert une population rom qui avait été maintenue dans l'état où elle se trouvait au début du XXe siècle. En partant vers l'Europe de l'Ouest, les Roms voudraient entrer dans le $\mathrm{XXI}^{\mathrm{e}}$ siècle, mais on les en empêche. On préfère les maintenir dans le siècle passé. Et je m'insurge contre cela.

Votre spectacle Django Drom, créé à l'occasion du centenaire de la naissance de Django Reinhardt, a été repris à la Cité de la musique pour l'ouverture de l'exposition consacrée au créateur du jazz manouche. Un orchestre hors du commun joue Django devant un film que vous avez réalisé spécialement. Comment a-t-il été conçu?

T. G. : La toile de fond du spectacle n'est pas un film, mais un peuple en marche. C'est un procédé de mise en scène qui n'existait pas. Généralement, les musiciens jouent en regardant l'écran pour accompagner les images. Mais là, ce sont les images qui accompagnent les musiciens. Elles apportent un surcroît d'émotion à la musique. L'orchestre, composé de 15 musiciens, est conduit par des "cadors" de la musique manouche: Biréli Lagrène, Stochelo Rosenberg, Hono Winterstein et Didier Lockwood, directeur musical de l'aventure. Installés devant un écran, ils interprètent la musique de Django Reinhardt. Les images qui défilent présentent toute la culture manouche, gitane, rom, à travers cinq siècles d'iconographie, des gravures de Jacques Callot (1592-1635) aux photos contemporaines, en passant par les cartes postales des années mille neuf cent. Toutes ces images sont en marche derrière les musiciens, le dos à l'écran et éclairés par un jeu de lumières. Le film bouge sur cette musique et l'on comprend l'évolution de ce peuple en mouvement, ainsi que l'arrivée de Django Reinhardt. Django Drom est un spectacle qui se lève! On en sort les yeux pleins d'images, les oreilles pleines de musique. On ne s'est pas fait bourrer le crâne avec des préjugés. La parole triche, mais la musique, elle, ne triche pas.

Comment abordez-vous la conception d'un spectacle comme Django Drom, en regard de celle d'un film?

T. G. : J'étais récemment au Festival des musiques sacrées du monde de Fès, où l'on m'a demandé de mettre en scène un spectacle sur Omar Khayam. Le spectacle est constitué de la même matière qu'un film. Il y a un scénario, une conduite et surtout une évolution entre le début, le milieu et la fin. La musique et les musiciens évoluent et les images, derrière eux, décrivent une émotion. La musique, elle aussi, crée l'émotion. Je joue sur les éclairages, la mise en scène pour tenter de lever la musique. J'ai abordé la mise en scène de Django Drom comme celle d'un film, avec des projecteurs et le décor mouvant sur l'écran. J'ai mis quatre mois à faire ce film d'environ une heure quarante. Certaines parties ont été tournées. Je me suis servi de documents que j'ai récupérés depuis une dizaine d'années à travers l'Europe : des cartes postales, des objets, des photos inédites... Je les ai filmés avec une caméra mouvante. On rentre dans les photos par un procédé conçu avec les gens avec qui je travaille. C'est comme du 3D, même si ça n'en est pas. Les objets et les gens bougent dans les photos. Et ils bougent en rythme avec la musique.

Le Boléro de Ravel était à l'origine du spectacle Django Drom. Comment cette idée a-t-elle évolué jusqu'à sa création sur scène?

T. G. : Il y a une quinzaine d'années, sur le tournage de Swing, j'étais avec Tchavolo Schmitt et Mandino Reinhardt. On se marrait ensemble à l'idée de faire un "Ali Baba et les 40 voleurs de poules". Et puis je me suis dit qu'un jour, ce serait bien de faire 
"Ali Baba et les 40 guitares" : un concert avec quarante guitares manouches. J'ai soumis cette idée à Dominique Delorme, alors directeur artistique des Nuits de Fourvière, et pour l'anniversaire de la naissance de Django Reinhardt il m'a proposé de monter ce concert. Le Boléro de Ravel est partie intégrante de l'idée des quarante guitares. Il y a dans cette œuvre une organisation qui vient de l'Espagne et de la transe. On trouve ce procédé de boucle qui revient et qui part dans les musiques tsigane, gitane, manouche et soufie. J'avais très envie de faire le Boléro de Ravel à la façon tsigane, avec des guitares.

Django et Ravel sont des légendes de l'art. Leur mort les a élevés au rang d'icônes. Or vous avez l'habitude de filmer des personnages très vivants. Comment s'est effectué le choix des musiciens de Django Drom?

T. G. : À travers sa musique et son parcours, Django a laissé un monument historique inimaginable. Peut-être l'héritage le plus positif du monde gitan. Pour cela, on devrait l'honorer, lui élever des statues !... Mais à l'inverse, son nom ne figurait nulle part à Paris, pas même à La Java ou dans les lieux de Pigalle qu'il fréquentait. C'est comme s'il n'existait pas, alors qu'il était connu dans le monde entier. Voilà bien la façon dont on traite les Roms, les Gitans, les Manouches en France et en Europe !... Il nous fallait lui rendre cet hommage. Et pour le faire, il fallait les plus grands : Biréli Lagrène et Stochelo Rosenberg. Bien sûr, derrière ces "cadors", il y a plein de jeunes qui sont de futurs "cadors". Ce qui me frappe dans cette musique des Roms, c'est la façon dont les enfants très jeunes s'y attaquent, comme l'a fait Biréli Lagrène, et comment la musique devient leur parole. En vous parlant, je lance des mots, Biréli lance des notes. La musique est dans son corps. Quel que soit le son que vous fassiez, il le reproduit à la guitare. Je suis en respect devant cette faculté qu'il a d'être traversé par la musique. Elle en devient quelque chose de magique, de spirituel. Derrière sa musique, il y a tout son sang, tout son peuple, tous ses parents... C'est ce que je vois quand il joue. Biréli n'est pas un musicien de conservatoire, il ne déchiffre pas la musique, c'est un musicien du vrai. Les Manouches jouent une musique plus archaïque, plus humaine, qui nous arrive plus vite.

Vous avez une relation fusionnelle avec la musique dans vos films. Qu'en est-il quand vous passez la main à des musiciens de la trempe de Didier Lockwood, Biréli Lagrène ou Stochelo Rosenberg?

T. G. : Je parle beaucoup avec les musiciens. Même s'ils improvisent et font des choses incroyables, parfois il y a des audaces qu'ils n'osent pas. Pour le Boléro de Ravel, Biréli se sentait coincé. Il ne voyait pas ce qu'il pouvait faire. Je lui ai dit: "Tout à l'heure, tu as joué instinctivement en suivant la musique, mais tu n'as pas joué le Boléro. Et c'est ça qui est bien !" C'est ainsi qu'il est entré dans le Boléro de Ravel, sans suivre la partition écrite. La guitare part dedans en improvisations manouches.

Vous aviez besoin d'un Lockwood pour assurer la direction musicale?

T. G.: Oui, il me fallait un constructeur de la musique. Pour ce spectacle qui fonctionne sur de la musique d'aficionados, il fallait quelqu'un qui assure une certaine rigueur. Didier Lockwood est un improvisateur incroyable. Élève de Stéphane Grappelli, il sait magnifiquement manier la musique de Django Reinhardt.

Comment appréhendez-vous l'interaction entre vos images et les musiciens en scène? Le cinéaste peut-il avoir la liberté d'improvisation qu'ont les musiciens ?

T. G. : Le montage est un délire. Il n'est pas fixe, parce que les images bougent au rythme de la musique. Les morceaux sont calibrés mais la musique peut se décaler, 
s'allonger selon l'interprétation. Mais même avec ces décalages, le rythme des images reste en harmonie avec elle. Les images baignent dans la musique manouche. Il y a un rythme de la caméra et des mouvements d'images.Il y a aussi des yeux, des cheveux de femmes, des routes, des paysages... Sur l'écran géant devant lequel les musiciens rivalisent de prouesses, défile le peuple rom en cohortes festives, en chariots, en regards, en volant voltigeant. Bohémien forgeron, danseuse au tambourin, compagnie vagabonde, Gypsies, Jitanos, Zigeuner, des tribus de Romanichels de tous âges et de toutes conditions, ours, roulottes, tubas, guitares emportent l'attention. Au rythme percutant des phrases de Django, le spectateur devient un voyageur, emporté dans cette migration sans fin qui est celle des nomades. Django Drom, machine à voyager bien au-delà de la musique dans l'histoire de ce peuple, ne sera plus joué sur scène. Tony Gatlif l'a annoncé à l'issue de la dernière représentation à la Cité de la musique. Sans doute ce magnifique spectacle est-il trop onéreux à produire... Espérons toutefois que sa musique a été fixée et qu'on pourra un jour en expérimenter une version cinématographique en film ou en DVD, afin que soit gardée la trace de ce splendide hommage.

\section{RÉSUMÉS}

Le Musée de la musique de Paris consacre une exposition à Django Reinhardt jusqu'en janvier 2013. Django Drom, spectacle créé par Tony Gatlif à l'occasion du centenaire de la naissance du génie de la guitare manouche, marquait son ouverture. Les talents de Didier Lockwood, Biréli Lagrène et Stochelo Rosenberg s'associaient à ceux d'une douzaine d'excellents artistes. Le jeu d'images mouvantes élaboré par le cinéaste en trame narrative du spectacle conférait une dimension bouleversante à l'œuvre de Django. 